\title{
ESTUDO DO PROCESSO DE RECICLAGEM DO POLIETILENO PARA A PRODUÇÃO DE BLENDAS *
}

Sérgio Luís de Moura Paiva Júnior ${ }^{1}$ Santino Louran Silvestre de Melo²

\section{Resumo}

A reciclagem de materiais provenientes de combustíveis fósseis é essencial para a redução do impacto desses materiais no meio ambiente. Utilizar materiais reciclados exige, não somente a reutilização direta do produto, mas a criação de processamento e técnica desses materiais no intuito de produzir materiais melhorados após o tempo de uso, justificando o desenvolvimento de tecnologias nesse âmbito. Esse trabalho de conclusão de curso foi executado no intuito de auxiliar nas técnicas utilizadas por uma empresa de reciclagem de polietileno para a produção de pellets reciclados. Foram avaliadas as propriedades mecânicas e espectroscópicas de cada uma das fases e os processos realizados para a produção do material final: uma blenda polimérica constituída de diferentes tipos de polietileno reciclado. As técnicas de caracterização utilizadas foram Ensaios de Tração para a avaliação da tensão máxima e módulo de elasticidade dos materiais, Microscopia Eletrônica de Varredura (MEV) para avaliar dimensionalmente a região de fratura dos polímeros, Infravermelho com Transformada de Fourier (FTIR) para identificar os grupos funcionais presentes nos polímeros analisados, TG/DTG para o estudo das propriedades térmicas das fases separadamente e da blenda e determinação do grau de cristalinidade por meio da densidade. Foi possível não só identificar as funções orgânicas do polietileno na técnica de espectroscopia de infravermelho, mas também analisar um pico desconhecido em um dos polietilenos envolvido na mistura da blenda, que pode se relacionar com o seu resultado obtido no ensaio de TG/DTG, o qual aponta uma maior porcentagem de resíduo após a degradação total da massa do mesmo.

Palavras-chave: Blendas; Polietileno; Reciclagem; Polímeros.

\section{STUDY OF THE RECYCLING OF POLYETHYLENE FOR THE PRODUCTION OF BLENDS}

\section{Abstract}

Recycle materials from fossil fuels is essential to reduce the impact of these materials on the environment. The use of recycled materials requires not only the direct reuse of the product, but the creation of processing and technique of these materials in order to improve better materials after the time of use, justifying the development of new technologies. This work was completed in order to assist in the techniques used by a polyethylene recycling company to produce recycled pellets. The mechanical and spectroscopic properties of each phase and the processes performed for the production of the final material were evaluated: a polymer blend composed of different types of recycled polyethylene. The characterization techniques used were Traction Tests for the evaluation of the maximum stress and modulus of elasticity of materials, Scanning Electron Microscopy (SEM) to dimensionally evaluate the fracture region of the polymers, Fourier Transform Infrared (FTIR) to identify the functional groups present in the analyzed polymers and TG / DTG for the study of the thermal properties of the phases separately and the blend. In view of the increase in technical knowledge about the characteristics of the recycled material, comparing with the mode of operation in which they are made, it is 
aimed to have more and assertive for the quality of the product. It was not only possible to identify the organic functions of the polyethylene in the infrared spectroscopy technique, but also to analyze an unknown peak in one of the polyethylenes involved in the blending mixture, which may be related to the result obtained in the TG / DTG assay. Which indicates a higher percentage of residue after the total degradation of the same mass.

Keywords: Blends; Polyethylene; Polymer; Recycling

1 Engenharia Metalúrgica, graduando, estudante, Departamento de Engenharia Metalúrgica e de Materiais, Universidade Federal do Ceará, Fortaleza, Ceará.

2 Ciência dos Materiais, doutorando, professor, Departamento de Engenharia Metalúrgica e de Materiais, Universidade Federal do Ceará, Fortaleza, Ceará. 


\section{INTRODUÇÃO}

A reciclagem de polímeros é uma das técnicas mais importantes para o desenvolvimento de tecnologias sustentáveis. O destino inadequado de resíduos sólidos pode afetar de maneira significativa o meio ambiente, fazendo com que esses produtos sejam vistos como "vilões". O processo de reciclagem é visto como um fator de desenvolvimento sustentável, fazendo com que essa técnica seja uma atividade fundamental na solução do destino desses resíduos, além dos fatores economicamente favoráveis nesse processo [1]. Os processos de reciclagem são, em geral, métodos que buscam a preservação do meio ambiente, pois não serão retiradas matérias primas do meio ambiente para a manufatura de novos produtos. Outro fator decisivo que sustenta os processos de reciclagem é a não contaminação ambiental após o descarte em aterros do produto utilizado [1].

\section{MATERIAIS E MÉTODOS}

Materiais utilizados, processo de obtenção das blendas e métodos de caracterização.

Para a realização dessa pesquisa de conclusão de curso, foram utilizados materiais reciclados de polietileno para a produção de blendas. Os materiais utilizados foram Polietileno Branco Reciclado, Polietileno Misto Reciclado e Polietileno Linear Reciclado para a produção de blendas. Dessas três matérias primas, foram confeccionados cinco tipos de blendas e avaliadas suas propriedades mecânicas. $O$ polietileno branco reciclado, polietileno de baixa densidade, material mais utilizado, por ser o principal material da blenda. O polietileno misto reciclado, devido ao processo rudimentar de reciclagem, contém outros tipos de polietilenos misturados, como o de alta densidade, ou até tintas, aditivos, adesivos e outros. Esse é o que possui maior proporção de impurezas. Por ser o classificado de menor custo e garantir uma queda na propriedade mecânica do material final, esse é, geralmente, misturado junto com uma proporção adequada de outro polímero. O polietileno linear reciclado, polietileno de baixa densidade linear, material de maior custo, usado para melhorar a qualidade de aderência e resistência mecânica da blenda na prática, compensando o uso do material misto.

As blendas confeccionadas nesse trabalho foram:

Blenda 1, matéria-prima que a empresa vende para a produção de sacola plástica reciclável, material conhecido como granulado de PEBD colorido, com composição de mistura do branco, misto e linear, nas seguintes proporções: $40 \%$ misto, $40 \%$ branco e $20 \%$ linear.

A blenda 2 é composta de $50 \%$ de polietileno linear reciclado e $50 \%$ de polietileno branco reciclado. A blenda 3 é composta de $20 \%$ de $\mathrm{PE}$ misto reciclado, $40 \%$ de PE branco reciclado e $40 \%$ de PE linear reciclado. Para a manufatura da blenda 4 , as proporções utilizadas foram de $30 \%$ de PE misto reciclado e $70 \%$ de PE branco reciclado. Na tabela abaixo, descrevemos cada um dos materiais e sua respectiva sigla para referência dos resultados. 
Tabela 1: Nomes e siglas dos materiais caracterizados.

\begin{tabular}{|l|l|}
\hline Nome & Sigla \\
\hline Polietileno Branco Reciclado & PBR \\
\hline Polietileno Misto Reciclado & PMR \\
\hline Polietileno Linear Reciclado & PLR \\
\hline $\mathbf{4 0 \%}$ Misto + 40\% Branco + 20\% Linear & B1 \\
\hline $\mathbf{2 0 \%}$ Misto + 40\% Branco + 40\% Linear & B2 \\
\hline $50 \%$ Linear + 50\% Branco & B3 \\
\hline $30 \%$ Misto + 70\% Branco & B4 \\
\hline
\end{tabular}

Fonte: Autor

O processo de obtenção das blendas se dá pelo método de extrusão de polímeros convencional, de matriz contínua, onde a etapa de lavar a matéria-prima é feita por um processo mais rudimentar em tanques de água, e não por um moedor. Primeiro é recolhido, classificado e separado o material reciclado, em polietilenos branco, misto e linear, depois passa pela etapa de trituração, limpeza, secagem parcial, aglutinação, secagem total, onde é preparado, misturando em proporções adequadas, pronto para ser fundido e homogeneizado na recuperação por extrusão, obtendo fios cilíndricos pela matriz contínua, imediatamente resfriados em água e granulado ou peletizado.

A partir de cada amostra, foi calculado sua densidade por método direto, fazendo 5 análises para cada um. Calcula-se então a cristalinidade a partir da densidade, que é um dado relevante para o entendimento das propriedades mecânicas. Placas para ensaio de tração foram feitas por termomoldagem. Retirou-se amostras de cada fratura do ensaio de tração para análise em MEV após ser metalizadas a vácuo. Uma placa de cada foi usada para determinar dureza shore D. Amostras dos polietilenos reciclados foram pulverizadas e prensadas, dissolvendo em $\mathrm{KBr}$ para análise espectroscópica de infravermelho. Por fim, pequenas quantidades das amostras dos polietilenos reciclados, junto com uma das blendas foram ensaiadas termicamente. 


\section{RESULTADOS E DISCUSSÃO}

Os ensaios de tração foram realizados para retornar os valores de Módulo de Elasticidade (E), Limite de Escoamento e Tensão Máxima. A figura 1 mostra os resultados dos ensaios de tração em gráficos apresentados em tensão VS deformação para cada um dos cinco corpos de prova produzidos e ensaiados.

Tabela 2: Resultados do ensaio de tração, ensaio de dureza, das medidas de densidade, dos calculos do grau de cristalinidade e da análise térmica.

\begin{tabular}{|c|c|c|c|c|c|c|c|c|c|}
\hline Siglas & $\begin{array}{c}\mathrm{E} \\
(\mathrm{MPa}) \\
(\sigma)\end{array}$ & $\begin{array}{l}\text { Tensão } \\
\text { Escoamento } \\
(\mathrm{Mpa})(\sigma)\end{array}$ & $\begin{array}{c}\text { Tensão } \\
\text { Máxima } \\
(\mathrm{Mpa}) \\
(\sigma)\end{array}$ & $\begin{array}{c}\text { Densidade } \\
\text { g/cm3 }\end{array}$ & $\begin{array}{c}\text { Grau } \\
\text { Cristalinidade } \\
\%\end{array}$ & $\begin{array}{l}\text { Dureza } \\
\text { Shore } \\
D(\sigma)\end{array}$ & $\begin{array}{c}\text { Máxima } \\
\text { Degradação } \\
\text { (으) }\end{array}$ & $\begin{array}{c}\text { Início de } \\
\text { Degradação } \\
\text { (으) }\end{array}$ & $\begin{array}{c}\text { Resíduo } \\
(\%)\end{array}$ \\
\hline$P B R$ & $\begin{array}{r}502,26 \\
\pm 13,25 \\
\end{array}$ & $2,46 \pm 0,064$ & $\begin{array}{c}7,14 \\
\pm 0,27 \\
\end{array}$ & 0,90 & 37,9 & $\begin{array}{c}37 \\
\pm 4,08 \\
\end{array}$ & 480 & 220 & 2,564 \\
\hline$P M R$ & $\begin{array}{l}323,28 \\
\pm 15,55 \\
\end{array}$ & $2,11 \pm 0,311$ & $\begin{array}{r}8,36 \\
\pm 0,37\end{array}$ & 0,92 & 53,1 & $\begin{array}{c}42 \\
\pm 1,60 \\
\end{array}$ & 470 & 225 & 0,9904 \\
\hline PLR & $\begin{array}{r}511,14 \\
\pm 32,02 \\
\end{array}$ & $2,01 \pm 0,118$ & $\begin{array}{c}5,64 \\
\pm 0,31 \\
\end{array}$ & 0,87 & 13,1 & $\begin{array}{c}39 \\
\pm 0,40 \\
\end{array}$ & 480 & 213 & 3,83 \\
\hline B1 & $\begin{array}{r}483,98 \\
\pm 48,72 \\
\end{array}$ & $2,43 \pm 0,176$ & $\begin{array}{r}8,96 \\
\pm 0,57 \\
\end{array}$ & 0,90 & 34,9 & $\begin{array}{c}32 \\
\pm 2,08 \\
\end{array}$ & & & \\
\hline$B 2$ & $\begin{array}{l}254,63 \\
\pm 14,67 \\
\end{array}$ & $2,14 \pm 0,136$ & $\begin{array}{r}7,40 \\
\pm 0,44 \\
\end{array}$ & 0,90 & 35,6 & $\begin{array}{c}39 \\
\pm 1,28 \\
\end{array}$ & & & \\
\hline B3 & $\begin{array}{l}343,67 \\
\pm 5,25 \\
\end{array}$ & $2,40 \pm 0,48$ & $\begin{array}{c}9,61 \\
\pm 0,48 \\
\end{array}$ & 0,91 & 43,7 & $\begin{array}{c}42 \\
\pm 0,80 \\
\end{array}$ & & & \\
\hline B4 & $\begin{array}{l}336,22 \\
\pm 31,56\end{array}$ & $2,42 \pm 0,48$ & $\begin{array}{c}8,82 \\
\pm 1,12\end{array}$ & 0,88 & 24,4 & $\begin{array}{c}38 \\
\pm 1,28\end{array}$ & & & \\
\hline
\end{tabular}

Das médias dos resultados obtidos, podemos observar que as tensões máximas decrescem na seguinte ordem: PLR, PBR e PMR, indicando que o polietileno linear possui maior resistência a tensão. Já o módulo de elasticidade que obteve maior valor, indica que esse deve ser utilizado com mais cautela para a produção de filmes, visto que sua deformação específica é menor que nos outros valores. Com os valores de cristalinidade e dureza, pretende-se observar uma tendência em relação à estas variáveis.

Os resultados apresentados acima mostram que a blenda 3 (B3) apresenta maior valor de tensão máxima, enquanto seu módulo de elasticidade é o menor dos três, mostrando que o resultado dessa blenda está de acordo com os resultados das matérias primas utilizadas, compostas por PBR e PLR, os polietilenos com maiores valores de tensão máxima e menores valores de módulo de elasticidade.

Podemos observar que o material base que apresenta maior dureza á o polietileno linear reciclado (PLR) e sua respectiva blenda (B3), com maior porcentagem do polietileno linear.

Os resultados da cristalinidade, mesmo que indiretos, colaboram com os resultados do ensaio de tração, indicando que a blenda com maiores valores de cristalinidade são as que possuem maiores porcentagem de matéria base com maior cristalinidade.

Da análise dos gráficos de TG/DTG retira-se a quantidade de resíduo que sobra após degradação e a temperatura de início de degradação do Polietileno Branco é de $220^{\circ} \mathrm{C}$, do Polietileno Linear é de $225^{\circ} \mathrm{C}$ e do Polietileno Misto é de $213^{\circ} \mathrm{C}$. 
Percebe-se que o polietileno linear possui menor valor de resíduo, que contribui com o método de obtenção dos materiais, já que o PLR é o polímero "melhor selecionado" dentre os outros polímeros. Já os polietilenos PBR e PMR apresentam maiores valores de massa residual.

\section{Infravermelho}

$\mathrm{Na}$ figura (1) abaixo, estão apresentadas as curvas de infravermelho com transformada de Fourier para os três tipos de polietileno reciclado e uma das blendas, confirmando assim que os materiais possuem os mesmos grupos funcionais e respectivamente os mesmos graus vibracionais.

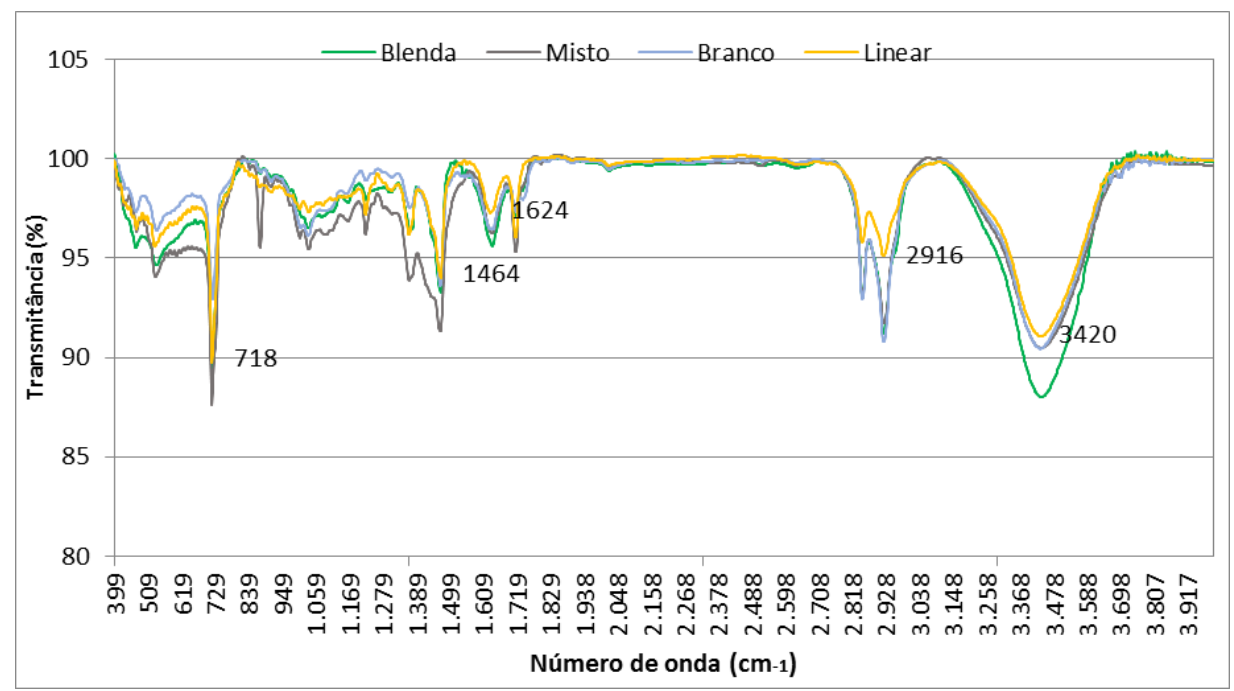

Figura 1: Curvas de infravermelho com transformada de Fourier para o B1, PMR, PBR e PLR.

$\mathrm{Na}$ figura acima, podemos identificar os picos referentes às bandas de absorção. As bandas de absorção referentes ao grupo funcional metila estão entre 1400 e $800 \mathrm{~cm}^{-}$ ${ }^{1}$, como pode ser observado nos gráficos para os quatro tipos de polietileno. No gráfico também é possível observar que os picos dos materiais coincidem, com exceção do PMR, que apresenta um pico aparente em $860 \mathrm{~cm}^{-1}$, indicando a presença de um $\mathrm{C}-\mathrm{H}$ fora do plano, com duplas ligações carbono-carbono, indicando que o processo inverso da polimerização foi iniciado, ou seja, uma degradação aparente.

\section{MEV}

Por fim, foram realizadas imagens em MEV do polietileno e suas respectivas regiões da fratura. As microscopias eletrônicas de varredura foram realizadas para cada uma das matérias prima base no intuito de observar no MEV a característica de cada uma das fraturas do material, com os indicativos de fratura rígida e fratura dúctil.
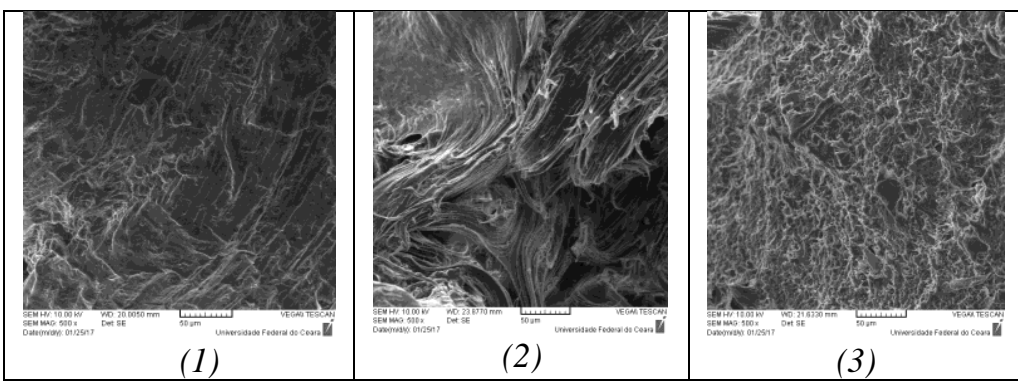
Figura 2: Imagem de 500x de aproximação do MEV da superfície da fratura do Polietilenos reciclados PBR (1), PLR (2) e PMR (3).

Foi possível observar arestas afiadas, indicando uma fratura frágil do polietileno branco reciclado (1), com a propagação rápida da trinca e deformação plástica pequena. Diferente dos outros, o PLR (2) teve maior elongação, maior esforço na ruptura e a ponta das arestas são curvas, determinando uma deformação dúctil no momento da fratura. Em (3) percebemos uma fratura do tipo frágil, com deformação plástica pequena, arestas afiadas paralelas ao eixo, perpendicular, portanto, ao esforço principal, resultantes da propagação rápida das trincas do material. [2].

\section{CONCLUSÃO}

Os resultados de ensaio de tração indicaram que o maior valor de tensão máxima era verificado para o polietileno linear reciclado, enquanto o maior valor de módulo de elasticidade era verificado para o polietileno misto. Portanto, para a rota de produção das blendas, as porcentagens de material devem ser direcionadas para as propriedades finais do compósito. Se busca-se um material mais resistente à tração, deve-se utilizar maior percentual de polietileno linear reciclado. Se for melhorar o módulo de elasticidade, deve-se optar pela utilização de maior percentual de polietileno misto reciclado. A dureza do polietileno reciclado linear se confirma por ter maior valor, consequentemente a blenda com maior porcentagem dele se confirma por tem maior dureza.

A cristalinidade obtida pelo método indireto de avaliação se tornou um adicional ao trabalho, apresentando uma boa ideia da adição de propriedades mecânicas com o aumento da cristalinidade das blendas produzidas e aumento da sua respectiva rigidez.

Com as curvas de termogravimetria foi possível identificar as temperaturas de início e final de degradação, além de observar que os maiores valores de resíduos foram identificados para o polietileno misto através da matéria que sobra após um tempo quando ultrapassado a temperatura de degradação, seguido do branco e por fim o linear, indicando assim que a quantidade de resíduos foi maior para os materiais ditos "menos selecionados".

O infravermelho com transformada de Fourier foi essencial para a identificação dos materiais como sendo constituídos de polietileno com seus grupos vibracionais específicos. Em um deles, o PMR, revelou-se um pico fora do plano que pode significar uma alteração química, oxidação ou reticulação, supostamente por conta de sucessivos ciclos de processamento ou solicitações de alta energia.

Com a microscopia eletrônica de varredura, pode-se distinguir para cada um dos materiais a sua respectiva fragilidade ou ductilidade, característicos dos padrões de fratura observados em MEV.

As conclusões tomadas a respeito do tipo de material caracterizado tiveram início no estudo da extrusão de polímeros assim como a extrusão do processo da empresa S.L.Plastic, onde tivemos oportunidades de implementar melhorias na manufatura como: 
Improvisar refrigeração na ponta do funil alimentador, início da extrusão, onde antes não havia, pois, a temperatura ali chega a degradar o material, o que estava ocasionando entupimento.

Trocar a tela, $25 \mathrm{~mm}$, de filtro de limpeza que fica junto à matriz para uma de $20 \mathrm{~mm}$, reduziu os problemas de impurezas, possibilitando visualizar uma nítida diferença na propriedade elástica da blenda.

\section{Agradecimentos}

Os autores agradecem à Universidade Federal do Ceará pelo suporte e estrutura, e a empresa S.L.Plastic, pela doação dos materiais reciclados e pelas blendas produzidas.

\section{REFERÊNCIAS}

1 Willian D. Callister, J. (2006). Fundamentos da Ciência e Engenharia de Materiais. Rio de Janeiro: LTC.

2 Canevarolo. (2006). Ciência dos Polímeros.

3 Blass, Arno. (1988). PROCESSAMENTO DE POLÍMEROS. FLORIANÓPOLIS: editora da UFSC.

4 Barbi, V.V. (1999). Estudo comparativo da morfologia cristalina de polietilenos obtidos por meio de diversos catalisadores. Tese de Doutorado - IMA /UFRJ.

5 Callister Jr, W. (1994). Materials Science and Engineering - An introduction . New York: Jhon Willey \& sons.

6 Danieletto, J. R. (1990). Tubos de Polietileno e Polipropileno . São Caitano do Sul: Medialdéa.

7 FELDMAN, D. E. (1996). A Synthetic Polymer Science and Technology. London: Chapman \& Hall.

8 Inoue, I. (1999). Influências da Incorporação de PEBDL em PEBD Industrial. Boletim Técnico OPP nº 1 - agosto, 1999.

9 Jr., S. V. (2007). Técnicas de Caracterização de Polímeros. São Paulo: Artliber Editora Ltda.

10 Norma ABNT - NBR 7456. (1982). Plástico - Determinação da dureza Shore.

11 Silva, Elaine A., e José M. M. Neto. (2016). "Possibilidades de melhorias ambientais no processo de reciclagem do polietileno." Polímeros.

12 Strapasson, R; Pereira, M.F.R; Sydenstricker, T.H.D; Amico, S.C. (2004) Análise da influência de múltiplas extrusões nas propriedades do polipropileno; II congresso de Termodinâmica Aplicada.

13 Torres, Amelia Angélica Ulloa. (2007). Envelhecimento fisico químico de tubulações de Polietileno de alta densidade empregadas em redes de distribuição de derivados de petroleo. Rio de Janeiro.

14 Wiebeck, Hélio, e Júlio Harada. (2005). PLÁSTICOS DE ENGENHARIA TECNOLOGIA E APLICAÇÕES. São Paulo: Artliber. 